\title{
A New Metadata Scheme for Multimedia and Intelligent Learning Objects
}

\author{
Jihad Chaker, Mohamed Khaldi
}

\begin{abstract}
The purpose of this contribution is to improve the interoperability of educational and multimedia metadata in the context of a new application profile based on the LOM standard, without affecting their educational purpose. our metadata analysis led to the creation of new elements and new categories by strengthening the semantic representation of pedagogical objects and the different structures of multimedia documents, namely: spatial, temporal and hypermedia structures, this proposal also includes the characteristics of description visual.

This contribution was essential given the absence of a metadata schema capturing multimedia and educational characteristics at the same time. the choice to gather descriptive elements based on the LOM standard, has proven to be wise since this standard is the most recognized and known in the field of eLearning. Throughout this article, we cite the advantages of pedagogical use of Multimedia, more specifically in eLearning. We then present intelligent learning environments on the one hand and educational objects on the other. Finally, we fix the new elements of our application profile, the latter is crowned with a semantic description in the form of an ontology.
\end{abstract}

Keywords : Multimedia content, Smart learning objects, Metadata interoperability, Application profile, Ontology, Multi-agent Systems, Ontology matching, E-learning standards.

\section{INTRODUCTION}

Metadata is used to describe the entities, and to promote interoperability between the various systems. The same applies to pedagogical objects: several metadata have been developed at different levels, including the schema, the application profile and the standard. This has a positive effect on the interoperability of metadata, particularly at the syntactic, linguistic and technical levels. However, the influence is relative given the various factors hindering interoperability, namely: the multiplicity and heterogeneity of systems, the multiplicity of protocols, the multiplicity of data formats, the diversity of metadata schemas, the diversity of vocabularies, ontologies and disciplines, The multiplicity of languages and the multiplicity of encoding formats.

Despite all these efforts, the problem of interoperability of pedagogical metadata persists, especially at the semantic level, which poses a problem of understanding the information exchanged between systems cooperating in the realization of a global task.

Revised Manuscript Received on December 30, 2019.

* Correspondence Author

Jihad CHAKER*, AbdelmalekEssaadi University, Faculty of Sciences,Tetouan, Morocco

Mohamed KHALDI, AbdelmalekEssaadi University, Faculty of Sciences,Tetouan, Morocco

(C) The Authors. Published by Blue Eyes Intelligence Engineering and Sciences Publication (BEIESP). This is an open access article under the CC BY-NC-ND license (http://creativecommons.org/licenses/by-nc-nd/4.0/)
To remedy this problem, we have moved to a new description of learning objects by adding semantic and multimedia features to better communicate with other systems.

\section{INTEROPERABILITY OF METADATA}

\section{A. Definitions}

Interoperability can be defined as the ability to communicate between two or more systems without any ambiguity. The report prepared by the committee CC: $\mathrm{DA}^{1}$ of the $\mathrm{ALCTS}^{2}$, specifies this communication in exchange for the information and the use of the information exchanged without any particular effort on the side of the systems [1]. The NISO ${ }^{3}$ institute uses the previous definition, adding as a primary criterion the minimal loss of content and functionality [2].

The ISO $^{4}$ proposes a definition that summarizes and details what is preceded, interoperability, is the ability to communicate, execute programs, or transfer data between different functional units in a way that does not require the user little or no knowledge of the unique characteristics of these units [3]. It covers both the data, their description, the services, the treatments, and the software tools that implement them.

In the context of the Web, metadata is information about web and other resources that are understandable by humans and treatable by software [4]. This information decries, explains, locates the resource and facilitates its research, use and management [2].

Through the analysis of metadata, we can underline three essential components: Instances, metadata schemas and description languages. The figure "Fig. 1"below illustrates these basic components of a scientific article.

${ }^{1}$ CC: DA: Committee on Cataloging: Description and Access.

${ }^{2}$ ALCTS: Association for Library Collections and Technical Services.

${ }^{3}$ NISO: National Information Standards Organization.

${ }^{4}$ ISO: International Organization for Standardization. 
A New Metadata Scheme for Multimedia and Intelligent Learning Objects

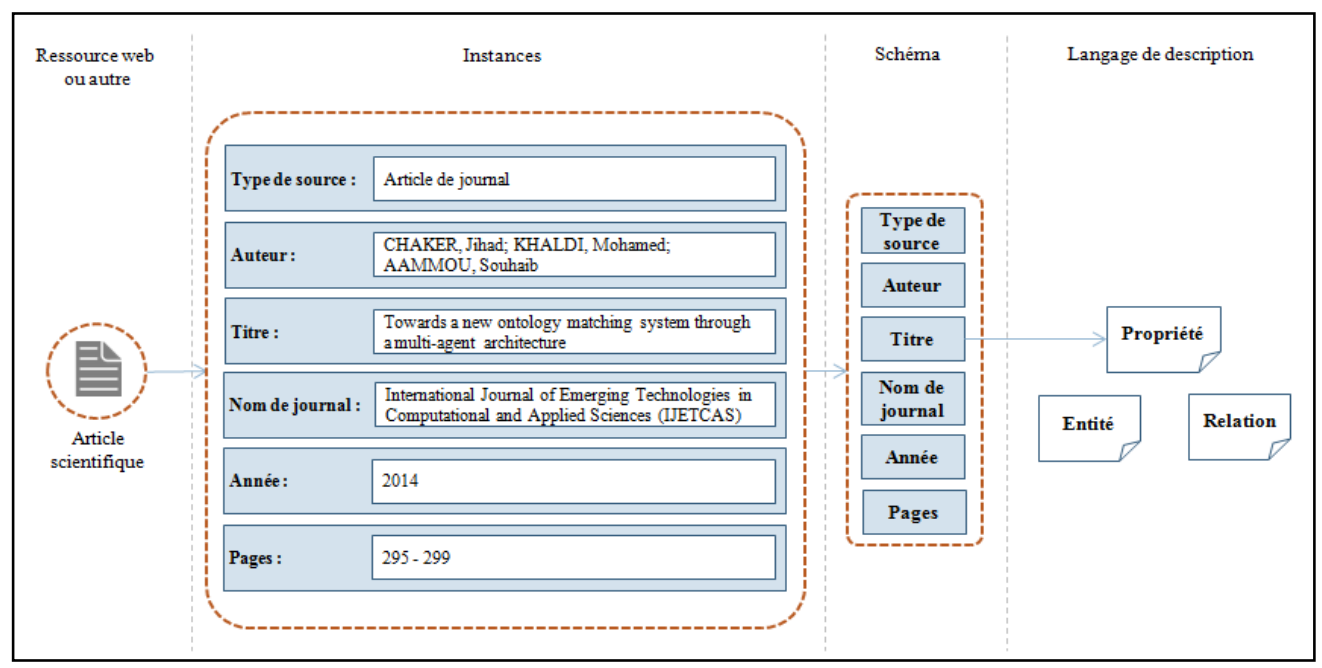

Fig.1: Basic components of metadata

Instances: Each metadata contains a set of attributes, each of them carries a value of the designated object;

Schemes of metadata: The preceding elements are organized and connected in a container called diagram which can be standardized later;

Description language: The attributes are typed as part of a schema definition language. The expressive power of languages is different from each other.

\section{B. Facets and factors preventing of interoperability of metadata}

There are several facets of metadata interoperability: technical, semantic, syntactic, and linguistic

Several studies conducted on the interoperability of metadata and the factors that hinder it, among others Moen [5] lists seven factors that are:

- Multiplicity and heterogeneity of systems;

- Multiplicity of protocols;

- Multiplicity of data formats;

- Diversity of metadata schemas,

- Diversity of vocabularies, ontologies and disciplines;
- Multiplicity of languages;

- Multiplicity of encoding formats.

Nilsson and colleagues [6].add two factors; the variety of abstract models on which metadata schemas are based and the identification of metadata elements.

\section{Ontology matching}

The knowledge translated by an ontology is conveyed using the following elements [7] : the concepts; relationships ; the functions ; the Axioms; el Instances. The "concepts", also called ontology terms or classes, correspond to the relevant abstractions of a segment of reality (the domain of the problem), selected according to the objectives we set ourselves and the intended application for ontology. "Relationships" translate the (relevant) associations between the concepts present in the analyzed segment of reality. "Axioms" are assertions, accepted as true, about domain abstractions translated by ontology. "Instances" constitute the extensional definition of ontology. They convey knowledge (static, factual) about the domain of the problem.

The figure "Fig. 2"below illustrates this notation for the example of the thesis report.

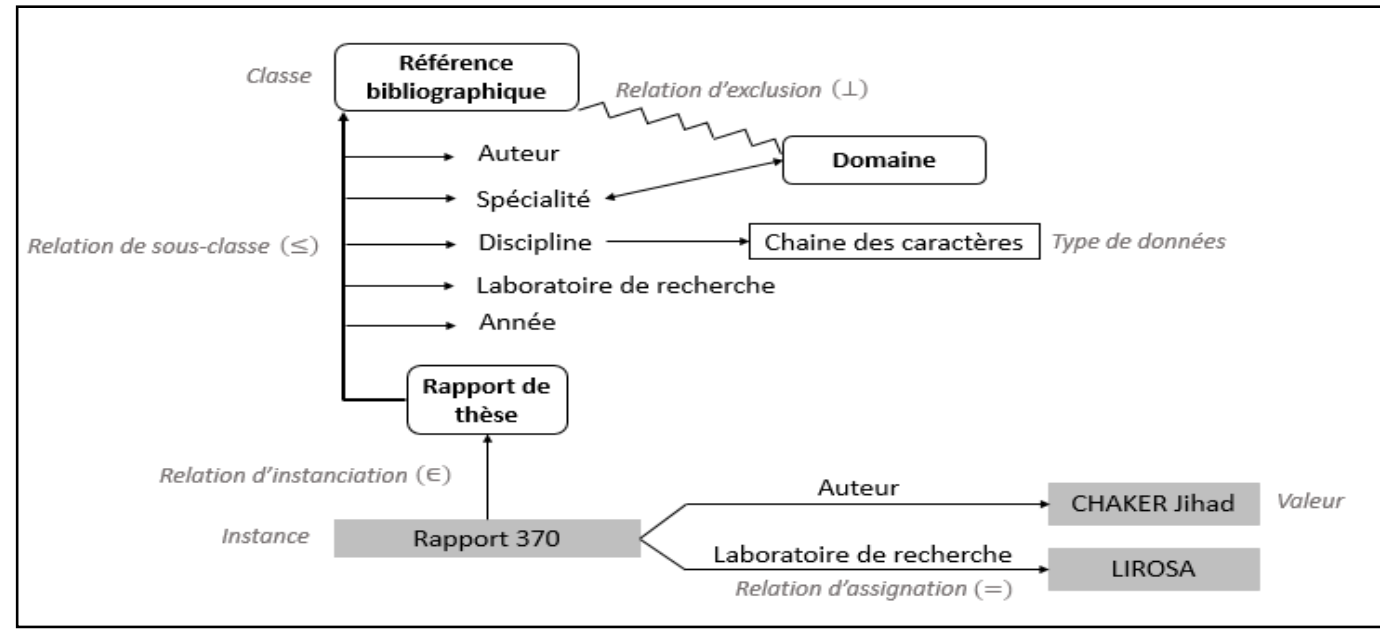

Fig.2 : Example of a graphical representation of ontologies 
Ontology matching is a function $\mathrm{f}$ which, applied to two ontologies o and o ', to an initial set of matches A, from a set of parameters $\mathrm{p}$ applying to the alignment techniques implemented. in the process and a set of external resources $\mathrm{r}$, produces a set of A 'matches between the two ontologies [8]: $\mathrm{A}^{\prime}=\mathrm{f}(\mathrm{o} ; \mathrm{o} ; \mathrm{A} ; \mathrm{p} ; \mathrm{r})$

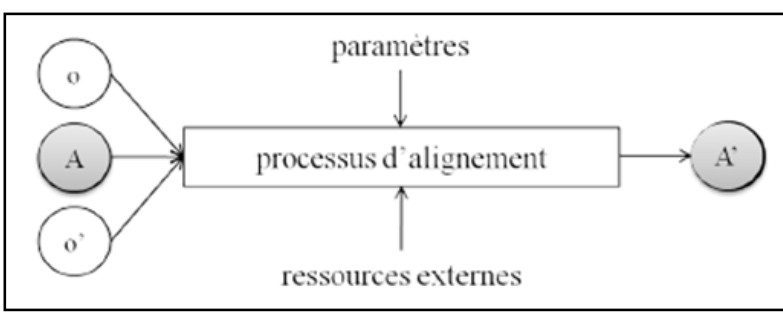

Fig.3 : Ontology alignment scheme according to [8]

\section{METADATA OF EDUCATIONAL OBJECTS AND MULTIMEDIA CONTENT}

\section{A. Definitions}

According to the IEEE LTSC group, an educational object is defined as "any entity, digital or otherwise, that can be used for teaching or learning" [9]. Strijker excludes the fact of non-numerical entities and states that pedagogical objects refer to digital entities that can be used and reused in different pedagogical and library situations" [10].

According to Bourda, pedagogical objects have the following properties [11]:

- Autonomy: Each educational object can be used independently of others.

- Reusability: An elementary pedagogical object can be used in multiple contexts and purposes. sets including for traditional courses.

- Indexing: Each educational object is provided with a description to find it easily.

To these properties are added the following properties addressed in the Gomez definition of educational resources [12]:

- Accessibility: Each educational object is provided with a description enabling it to be found, identified and easily accessed.

- Sustainability: An educational object must be able to cope with technological change by minimizing re-engineering or redevelopment.

- Interoperability: An educational object must be able to be used in different technological environments.

According to [13] in his report "Multimedia and semantic Web at the service of learning". A multimedia product can therefore be defined as a combination of digital media. Discreet (text, still images) and continuous (animated images, sound), synchronized and linked so as to allow the user to act on the progress of the application and to choose its itinerary of consultation (interactivity), and diffusable on telecommunication networks or any other digital medium (CD, DVD and future media).

In what follows, we list the various documentary structures most cited in the literature:

The logical structure allows a breakdown of the information from a hierarchical point of view, [14]specifies that the hierarchy must be according to a more or less fine decomposition principle. This mechanism imposes unambiguously identifying the information granules composing the document.

The physical structure defines the positioning and organization of the physical elements on a presentation medium.

- Aggregation: Pedagogical objects can be grouped into

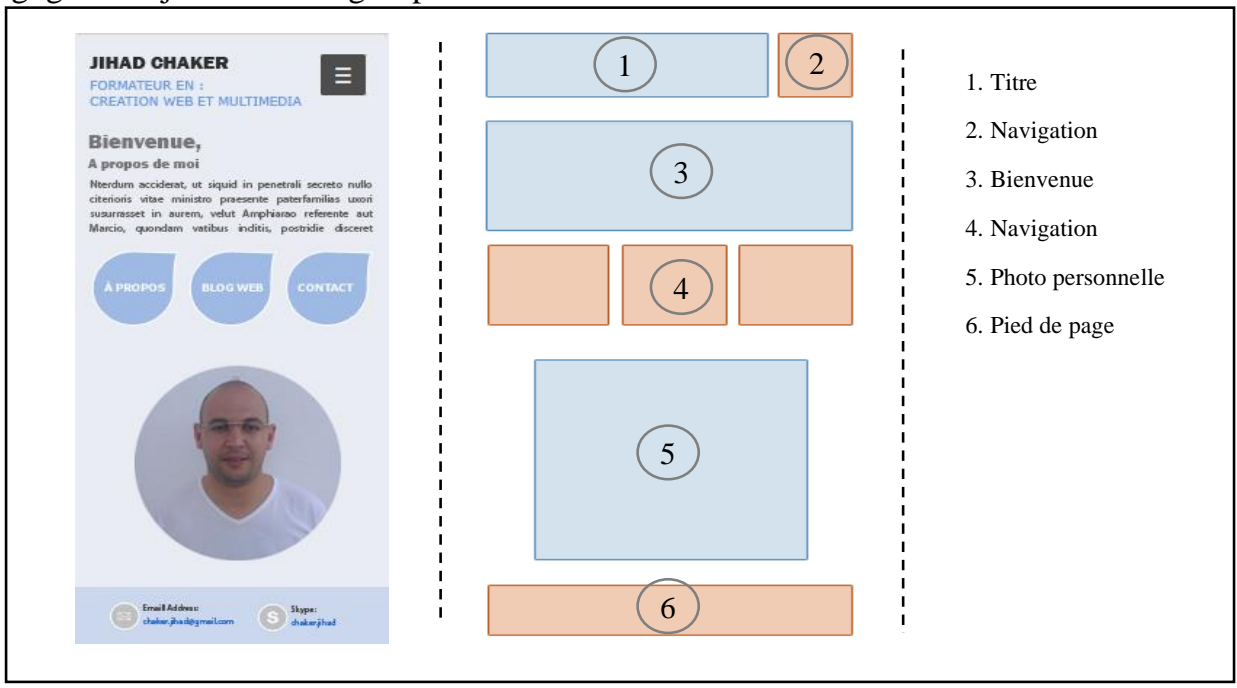

Fig.4: Example of physical structure

The spatial structure concerns the arrangement of multimedia objects in a 2D or 3D space, by defining their inter and intra-object positions. 


\section{A New Metadata Scheme for Multimedia and Intelligent Learning Objects}

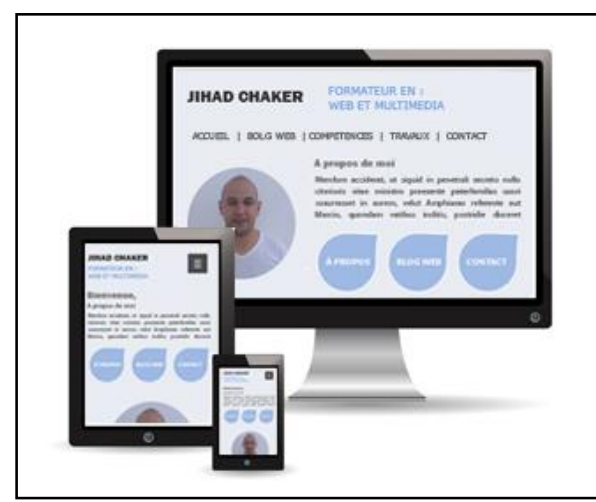

Fig.5 : Example of spatial structure

The hypermedia structure concerns the links between the documents or between the objects of the same document.

The semantic structure is a designation frequently used to identify structures that explain information about the meaning conveyed by different parts of a content [15].

The temporal structure ensures the synchronization of the multimedia objects in time. Below is Figure "Fig. 6" relationships for structuring the content of an audiovisual sequence based on the time axis. In what follows, an example of the temporal structure of a multimedia document created under Adobe Professional Flash.

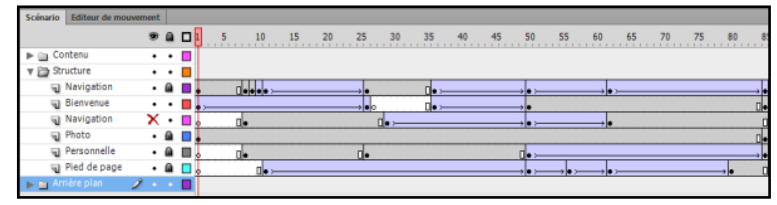

Fig.6: Example of the time structure in Adobe Professional Flash

The hypermedia structure concerns the links between the documents or between the objects of the same document.

The semantic structure is a designation frequently used to identify structures that explain information about the meaning conveyed by different parts of a content [15].

\section{B. Metadata of educational objects}

\section{- $\quad$ AICC specification}

AICC "Guidelines for CMI Interoperability" was the first widely adopted specification for interoperability between e-learning content and LMS. "AICC" also refers to the specification most widely adopted by AICC. It has as main features: Standardize communication between the content and the LMS; Manage the loading of content into an LMS; Adapt the pedagogical content according to the learner.

\section{- IMS specification}

Among the projects of the IMS we find:

○ The IMS "Content Packaging";

○ The IMS "Question and Test Interoperablility"

○ The IMS "Learning Object Metadata"

○ IMS "Learner Information Packaging"

○ The IMS "Enterprise Interoperability"

\section{- Standard LOM}

The LOM is largely based on the work initiated by the ARIADNE Foundation since 1997. It is currently the most used to promote the sharing and reuse of educational objects.
His metadata schema includes 80 hierarchical elements, grouped into nine categories. In the following we present the detail of the LOM.

Table- I: LOM categories

\begin{tabular}{|l|l|}
\hline Category & Description \\
\hline 1. General & $\begin{array}{l}\text { General context-independent characteristics that } \\
\text { describe the resource as a whole }\end{array}$ \\
\hline 2. Life Cycle & Life Cycle Characteristics \\
\hline $\begin{array}{l}\text { 3. } \\
\text { Meta-Metadata }\end{array}$ & $\begin{array}{l}\text { Characteristics of the description itself (specific to the } \\
\text { recording of metadata). }\end{array}$ \\
\hline 4. Technical & $\begin{array}{l}\text { Technical requirements in terms of browser, operating } \\
\text { system, or features. }\end{array}$ \\
\hline 5. Educational & Pedagogical characteristics of a resource. \\
\hline 6. Rights & Characteristics expressing the conditions of use \\
\hline 7. Relation & $\begin{array}{l}\text { Characteristics expressing links with other } \\
\text { educational resources by specifying the nature of the } \\
\text { relationship }\end{array}$ \\
\hline 8. Annotation & $\begin{array}{l}\text { Annotations or comments on the educational use of } \\
\text { the resource }\end{array}$ \\
\hline $\begin{array}{l}\text { 9. } \\
\text { Classification }\end{array}$ & $\begin{array}{l}\text { Characteristics of the resource described by entries in } \\
\text { classification systems. }\end{array}$ \\
\hline
\end{tabular}

\section{- LOM application profiles}

There are a number of application profiles based on the LOM that meet certain specific needs, including: LOMFR, SupLOMFR, Normetic ...

\section{- MLR standard}

The result of a long process of adoption by consensus, the new MLR teaching resource description standard is based on two widely adopted standards, the Dublin Core and the LOM.

\section{- SCORM specification}

The SCORM is the result of a standardization process between American governmental, academic and industrial actors. In 1997, the American Defense Department's Advanced Distributed Learning (ADL) project brought together a federation of heterogeneous actors including Ariadne, AICC, IEEE and IMS. It attempts to synthesize these different systems for one simple purpose: to make it a "cost effective" standard, ie easy to use and allowing the reuse of resources.

\section{Description of multimedia content based on the MPEG-7 standard}

The main goal of MPEG-7 is to ensure interoperability between systems and applications used in the production, management, distribution and consumption of multimedia content descriptions. These descriptions will help users or applications at the level of identification, extraction and filtering of audio-visual information. Examples of applications include the selection of radio and television media, digital libraries, multimedia directory services, and multimedia publishing. 


\section{THE PROPOSED METHODOLOGY}

\section{A. The place of multimedia in the field of e-learning}

It is undeniable that the integration of multimedia captures the attention of learners, or even offers them a better experience in the field of education without altering the educational objectives of the content.

Indeed, recent technologies improve the efficiency of instructional design, since they provide broad access to multimedia tools. However, e-learning professionals take it into consideration. In other words, the pedagogical use of multimedia is changing pedagogical scenarios, as it simplifies both knowledge and makes online courses more captivating.

\section{B. Elements of the metadata application profile}

Instead of proposing a new metadata to describe intelligent pedagogical objects, we proposed to create an application profile of the LOM standard, since this standard is the international reference for describing pedagogical objects.

The information is distributed, for the application profile, in 11 categories "Fig. 7". New items proposed in blue.

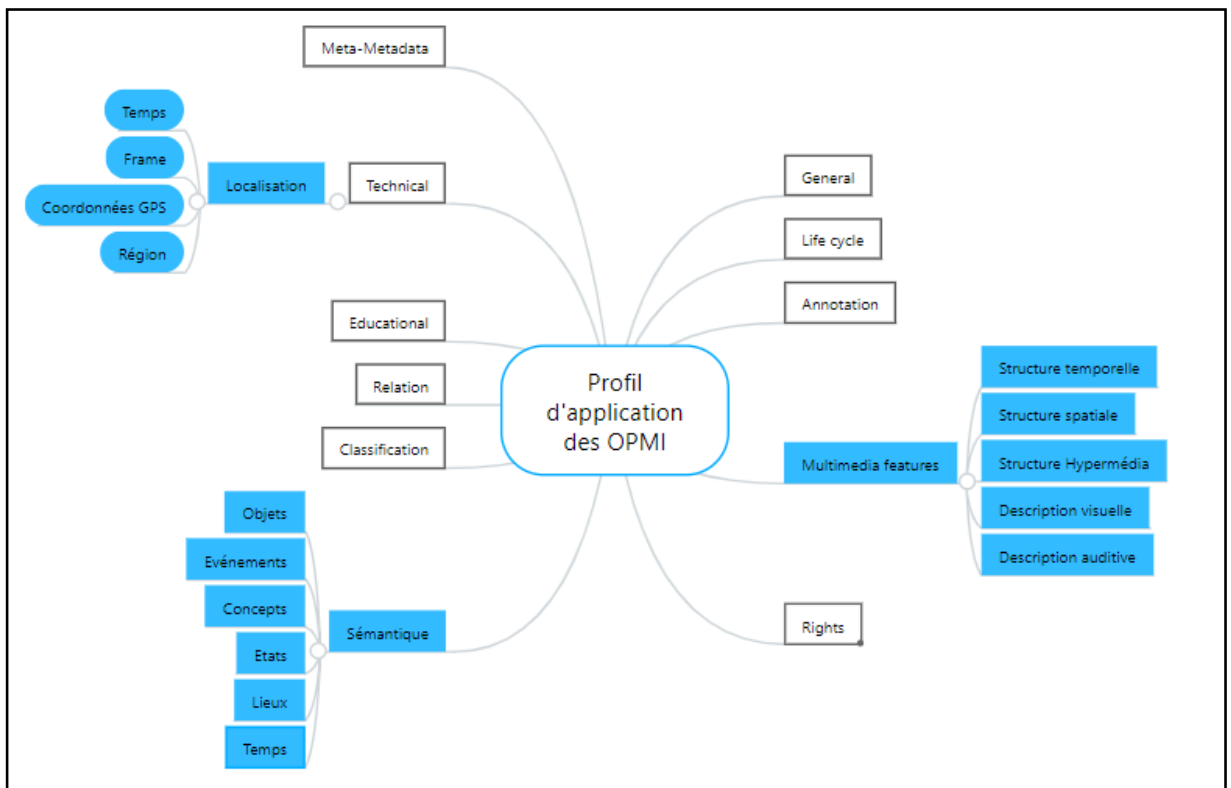

Fig.7 : elements of the proposed application profile

\section{Improved interoperability of educational and multimedia metadata}

Research in the field of ontology alignment has shown the effectiveness of combining several matches in parallel or successive. Therefore we did a benchmarking of the different matchers used as well the similarity measures.

The proposed general process has four stages, the first is to apply a set of techniques for the ontologies to be ready to be aligned, the second stage is composed of a series of similarity computations, precisely at the syntactic, lexical, structural and semantics. These similarities are based on a set of functions called matchers, the result of this process is to generate candidate matches that will be proposed to the filtering process, to validate or cancel them. Afterwards, a series of structural and semantic similarities will be applied, then the step filtering and validation by the expert or automatic. All of these steps are illustrated in the figure "Fig. 8 ".

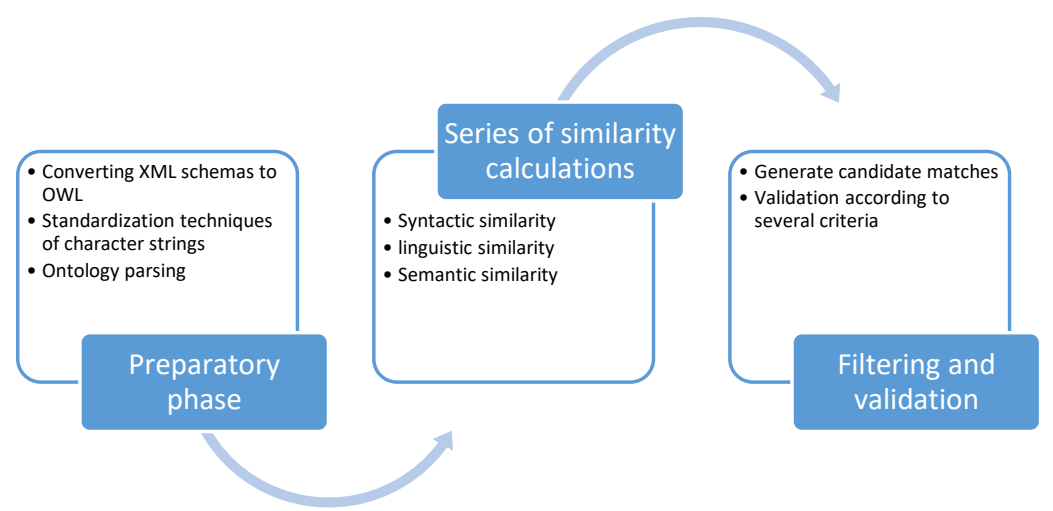

Fig.8: general process of the method 


\section{A New Metadata Scheme for Multimedia and Intelligent Learning Objects}

\section{EXPERIMENTED RESULTS}

We focused on semantic metadata interoperability using the ontology alignment approach which consists of aligning semantic metadata concepts to facilitate communication between intelligent systems.

Below are the results of the concepts of semantic metadata using the methodology described above.

Table- II: Application on educational metadata and Multimedia : MPEG-7 Path and LOM Meta-data Path

\begin{tabular}{|l|l|}
\hline \multicolumn{1}{|c|}{ MPEG-7 Path } & \multicolumn{1}{c|}{ LOM Meta-data Path } \\
\hline $\begin{array}{l}\text { MediaInformation.MediaI } \\
\text { dentification.EntityIdentifi } \\
\text { er }\end{array}$ & $\begin{array}{l}\text { MediaInformation.MediaId } \\
\text { entification.EntityIdentifier }\end{array}$ \\
\hline general.identifier & general.identifier \\
\hline $\begin{array}{l}\text { CreationInformation.Creat } \\
\text { ion.Title }\end{array}$ & $\begin{array}{l}\text { CreationInformation.Creati } \\
\text { on.Title }\end{array}$ \\
\hline general.title & general.title \\
\hline $\begin{array}{l}\text { CreationInformation.Class } \\
\text { ification.Language }\end{array}$ & $\begin{array}{l}\text { CreationInformation.Classi } \\
\text { fication.Language }\end{array}$ \\
\hline general.language & general.language \\
\hline
\end{tabular}

Table- III: Application on educational metadata and Multimedia : MPEG-7 Path and SCORM Meta-data Path

\begin{tabular}{|l|l|}
\hline \multicolumn{1}{|c|}{ MPEG-7 Path } & SCORM Meta-data Path \\
\hline Segment & Segment \\
\hline Asset & Asset \\
\hline Package DS & Package DS \\
\hline Package & Package \\
\hline Collection & Collection \\
\hline Manifest & Manifest \\
\hline
\end{tabular}

\section{CONCLUSION}

This chapter introduces a new metadata schema for multimedia and intelligent learning objects by clarifying the place of multimedia in the field of e-learning and pedagogical objects in intelligent learning environments. Next Application Profile for Multimedia and Smart Learning Objects by Focusing The choice of the Application Profile, the metadata application profile elements and the application profile in OWL.

The next work aims to evaluate the performance of our algorithm, passing a series of tests using some tests provided in the Benchmark database made available to the international community by the competition EON, also a comparison with other methods.

\section{REFERENCES}

1. CC:DA. (2000). Task Force on Metadata. Accessed September 14, 2019, onhttp://www.libraries.psu.edu/tas/jca/ccda/tf-meta6.html

2. NISO. (2004). Understanding Metadata. Accessed September 14, 2019,

onhttp://www.niso.org/publications/press/UnderstandingMetadata.pdf

3. ISO/IEC 2382-1. (1993). Information Technology - Vocabulary - Part 1: Fundamental terms.

4. Berners-Lee, T. (1997, Janvier 6). Metadata Architecture. Accessed September 14 ,

2019, onhttp://www.w3.org/DesignIssues/Metadata.html

5. Moen, W. E. (2004, Mai 20). Metadata Interaction Integration and Interoperability. NISO Workshop: Metadata Practices on the Cutting Edge.

6. Nilsson, M., Naeve, A., Duval, E., Johnston, P., \&Massart, D. (2008). Harmonization of metadata standards. Network of Excellence in Professional Learning PROLEARN.

7. Pinto, H. S., Gómez-Pérez, A., \& Martins, J. P. (1999). Some issues on ontology integration. Proceedings of the IJCAI-99 Workshop on Ontologies and Problem-Solving Methods: Lessons Learned and Future Trends. Stockholm.

8. Shvaiko, P., \&Euzenat, J. (2013). Ontology matching: state of the art and future challenges. IEEE Transactions on Knowledge and Data Engineering (TKDE), 25(1), 158-176.

9. IEEE-LTSC. (2002). Draft standard for learning object metadata.

10. Strijker, A. (2004). Reuse of learning objects in context: Human and technical aspects. University of Twente.

11. Bourda, Y. (2001). Objets pédagogiques, vous avez dit «objets pédagogiques»? Cahiers GUTenberg, 8254

12. Gómez-Pérez, A. (1996). Towards a framework to verify knowledge sharing technology. Expert Systems with Applications, 11(4), 519-529.

13. Roxin, I. (2003). Multimédia et Web sémantique au service de l'apprentissage.

14. Mbarki, M. (2008). Gestion de l'hétérogénéité documentaire: le cas d'un entrepôt de documents multimédia (Doctoral dissertation, Université de Toulouse, Université Toulouse III-Paul Sabatier).

15. Chatti, N. K. (2007). MultiX: an XML-based formalism to encode multi-structured documents. In Proceedings of Extreme Markup Languages.

\section{AUTHORS PROFILE}

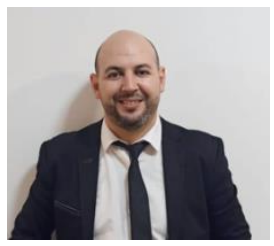

present on 5 continents

(E-mail: chaker.jihad@gmail.com).

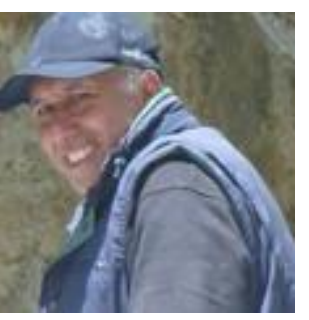

Mohamed KHALDIis a professor at the TheÉcole Normale Supérieure at AbdelmalekEssaadi University, Laboratory of Informatics, Research Operational and Statistic Applied (LIROSA) at Faculty of Sciences, AbdelmalekEssaadi University. Tétouan, Morocco, member of the scientific committee at ENS Tétouan, Coordinator of the Specialized Master in Multimedia Pedagogical Engineering ENS Tétouan, Member of the National Board of the International Association of University Pedagogy (AIPU), Morocco Section and Delegate of the section AbdelmalekEssaadi of the International Association of University Pedagogy (AIPU) section Morocco. His current research focuses on : e-learning, Field of the integration of the computer tool in the teaching, Field of discipline didactics and Production of teaching aids. (E-mail: medkhaldi@yahoo.fr). 